\title{
LsrR-binding site recognition and regulatory characteristics in Escherichia coli Al-2 quorum sensing
}

\author{
Ting Xue ${ }^{1}$, Liping Zhao ${ }^{1}$, Haipeng Sun ${ }^{1}$, Xianxuan Zhou ${ }^{1}$, Baolin Sun ${ }^{1}$ \\ ${ }^{I}$ Hefei National Laboratory for Physical Sciences at Microscale and School of Life Sciences, University of Science and Technology \\ of China, Hefei 230027, China
}

In quorum sensing $(\mathrm{QS})$ process, bacteria regulate gene expression by utilizing small signaling molecules called autoinducers in response to a variety of environmental cues. Autoinducer 2 (AI-2), a QS signaling molecule proposed to be involved in interspecies communication, is produced by many species of gram-negative and gram-positive bacteria. In Escherichia coli and Salmonella typhimurium, the extracellular AI-2 is imported into the cell by a transporter encoded by the $l s r$ operon. Upstream of the $l s r$ operon, there is a divergently transcribed gene encoding LsrR, which was reported previously to repress the transcription of the $l s r$ operon and itself. Here, we have demonstrated for the first time that LsrR represses the transcription of the $l s r$ operon and itself by directly binding to their promoters using gel shift and DNase I footprinting assays. The $\beta$-galactosidase reporter assays further suggest that two motifs in both the $l s r R$ and $l s r A$ promoter regions are crucial for the LsrR binding. Furthermore, in agreement with the conclusion that phosphorylated AI-2 can relieve the repression of LsrR in previous studies, our data show that phosphoAI-2 renders LsrR unable to bind to its own promoter in vitro.

Keywords: quorum sensing, LsrR, AI-2

Cell Research (2009) 19:1258-1268. doi: 10.1038/cr.2009.91; published online 28 July 2009

\section{Introduction}

Quorum sensing (QS) is a process of cell-cell communication that bacteria use to regulate specific physiological functions in response to the fluctuations of the population density in a community $[1,2]$. QS bacteria secrete small, diffusible signaling molecules called autoinducers that accumulate in the external environment. When the concentration of the autoinducers reaches a threshold, an alteration of gene expression is induced, allowing the bacteria to adopt behaviors that are only productive when the bacteria are working together as a group [3, 4]. Among many different mechanisms for QS by bacteria, one is shared by both gram-negative and gram-positive bacteria, involving the production of autoinducer 2 (AI-2) [5-10].

AI-2 QS system was first identified in the marine

Correspondence: Baolin Sun

Tel: 86-551-3606748; Fax: 86-551-3607438

E-mail: sunb@ustc.edu.cn

Received 8 February 2009; revised 17 March 2009; accepted 15 May 2009; published online 28 July 2009 bacterium Vibrio harveyi as a part of a complex multilayered QS system responsible for regulating bioluminescence and other virulence-associated traits $[4,11]$. Subsequently, AI-2 production was verified in a large number of species and the AI-2 QS system was recognized to be involved in the regulation of a range of functions in diverse bacteria $[6,7,10,12-14]$. In contrast to other autoinducers that are specific for a narrow range of organisms, the widely conserved AI-2 has been hypothesized to be a universal language for interspecies communication $[6,9,10,13,15]$. In every case, AI-2 is synthesized by LuxS, which functions in the pathway for metabolism of S-adenosylmethionine (SAM), a major cellular methyl donor. In a metabolic pathway known as the activated methyl cycle, SAM is metabolized to Sadenosylhomocysteine, which is subsequently converted to adenine, homocysteine, and 4,5-dihydroxy-2,3-pentanedione (DPD, the precursor of AI-2) by the sequential action of the enzymes Pfs and LuxS $[10,16]$. DPD is a highly reactive product that can rearrange and undergo additional reactions, suggesting that distinct but related molecules derived from DPD may be the signals that different bacterial species recognize as AI-2. Experimental 
evidence showed that in $V$. harveyi, AI-2 is $(2 S, 4 S)$-2methyl-2,3,3,4-tetrahydroxytetrahydrofuran-borate $(S$ THMF-borate); and in E. coli and S. typhimurium, AI-2 is $(2 R, 4 S)$-2-methyl-2,3,3,4-tetrahydroxytetrahydrofuran (R-THMF) [10, 16-22].

Although the pathway for AI-2 production is widely conserved among bacteria, the mechanisms of AI-2 detection and signal transduction are specific and have only been established in a few species such as $V$. harveyi, $V i$ brio cholerae, S. typhimurium, and E. coli. In V. harveyi, $\mathrm{AI}-2$ detection and transduction rely on a two-component system similar to that of the QS system in gram-positive bacteria [11]. Boron-AI-2 is recognized by a soluble periplasmic protein that interacts with cytoplasmic response regulator proteins, thus triggering a sensor-kinase couple to finally control the expression of the luciferase structural operon (luxCDABE) [4, 21, 23, 24].

In $E$. coli and $S$. typhimurium, $\mathrm{AI}-2$ is required to be imported into the cell for gene expression control. In $E$. coli and S. typhimurium, extracellular AI-2 accumulates in the exponential phase, but decreases drastically upon entry into the stationary phase. The rapid disappearance of AI-2 is due to its import by the $l s r$ transporter $[19,25$ 28]. In E. coli, the $l s r$ operon is comprised of six genes lsrACDBFG (Figure 1). The first four genes, lsr $A C D B$, encode components of the ATP-binding cassette transporter, and the remaining two, $l s r F G$, are proposed to be required for the modification of AI-2 following internalization $[28,29]$.

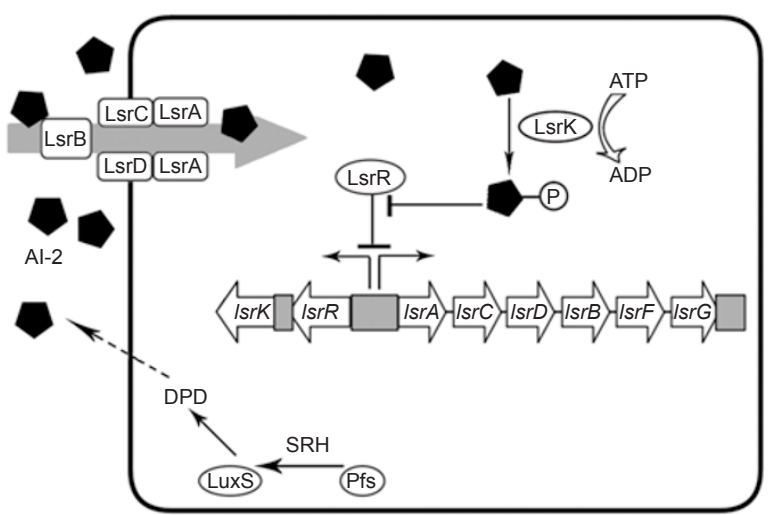

Figure 1 Model for regulation, transportation, and modification of Al-2 by the Lsr proteins in E. coli. Al-2 is synthesized by LuxS and accumulates extracellularly. The Al-2 uptake repressor LsrR represses the Isr operon (comprised of IsrACDBFG) and the IsrRK. Basal expression of the LsrACDB transporter allows some Al-2 to enter the cytoplasm, where it is phosphorylated by LsrK. Phospho-Al-2 has been reported to bind to LsrR and relieve its repression effect on the Isr transporter genes, thus stimulating additional $\mathrm{Al}-2$ uptake.
The regulatory network for AI-2 uptake is comprised of two other important components, $l s r R$ and $l s r K$, both of which are located adjacent, but divergently transcribed from the $l s r$ operon (Figure 1). LsrR is the repressor of the $l s r$ operon and itself. LsrK is a kinase responsible for converting AI-2 to phospho-AI-2, which is required for relieving LsrR repression. Functions of LsrR and LsrK can be partially revealed using $l s r K$ and $l s r R$ mutants. In $l s r R$ mutants, the LsrACDB transporter is produced, and extracellular AI-2 is continuously imported into the cell (cytoplasmic AI-2). In lsrK mutants, the expression of $l s r$ is repressed, and AI-2 remains in the supernatant (extracellular AI-2) [25-28, 30, 31]. Since LsrR contains a helix-turn-helix (HTH) DNA-binding domain, it was hypothesized that LsrR represses the expression of $l s r$ operon and itself by binding to their promoter regions [26, $29,32]$. However, no evidence of their direct binding has been shown yet. It has also been postulated that phosphoAI-2 binds to LsrR and inactivates it to derepress the transcription of $l s r[25,26]$. Although a previous study has revealed that phospho-AI-2 can bind to LsrR directly using in vitro assays [29], whether it is the same case in vivo and the detailed mechanism by which phospho-AI-2 derepresses the $l s r$ transcription still remain obscure. In addition, it is interesting that another previous study has suggested that LsrR and LsrK may serve as global regulators working on genes other than the $l s r$ operon, and hypothesized that LsrR is a QS regulator that can regulate the transcription of a variety of genes after binding to phospho-AI-2 [32]. Taking all these into account, it is important to explore the precise LsrR-binding mode.

In this study, we provide evidence for direct LsrR binding to the $l s r$ promoter and the promoter of its own by gel shift assays. DNase I footprinting assays show that LsrR binds to a region between -20 and -49 with respect to the $l s r R$ promoter. In addition, gel shift data also show that phospho-AI-2 prevents LsrR and the $l s r R$ promoter from forming a complex, providing in vitro evidence that phospo-AI-2 can alleviate the LsrR-mediated repression.

\section{Results}

\section{LsrR binds to lsrA and lsrR promoters}

LsrR has been revealed to be able to repress transcription from the $l s r A$ and $l s r R$ promoters, with deletion of $l s r R$ resulting in maximal transcription of the $l s r$ operon and the $l s r R$ gene in S. typhimurium and E. coli [26, 31]. Since LsrR contains a predicted HTH DNA-binding domain, it has been suggested to repress the transcription of the $l s r$ operon and itself by directly binding to their promoters. In order to determine whether LsrR can bind to the $l s r A$ and $l s r R$ promoter regions, we carried out gel 
shift assays. His6-tagged LsrR was overexpressed in $E$. coli BL21 (DE3) and purified using Ni-NTA affinity resin. Two DNA fragments p-lsrR (119 bp from -1 to -119 of the $l s r R$ translation start site) and p-lsrA (129 bp from -1 to -129 of the $l s r A$ translation start site) were PCRamplified and DIG-labeled (Figure 2A) for use as the probes. Results show that LsrR specifically binds to both $l s r A$ and $l s r R$ promoter regions. As shown in Figure 2B, from lane 2 to 5 , two bands are observed. The lower one is the free labeled probes present in molar excess, and the higher one represents a protein-DNA complex. As the amount of added LsrR protein increased from 5 to 40 pmol (the final concentration was from 0.5 to $4 \mu \mathrm{M}$ ), the amount of the complex formed increased and the amount of free probes decreased correspondingly. For control, no LsrR was added in lane 1 and 10-fold unlabeled fragments were used as specific competitors in lane 6 , and no protein-DNA complex was formed under these two conditions. We also used a random DNA sequence as the probe for the negative control, and the results show that LsrR cannot bind to this random fragment (data not shown). These data indicate that LsrR can specifically bind to the $l s r A$ and $l s r R$ promoter regions in vitro.

Phosphorylated AI-2 causes LsrR to relieve its binding to the promoters in vitro

In S. typhimurium and E. coli, LsrK can phosphory- late AI-2, and phospho-AI-2 is thought to be the signal molecule that activates transcription of the $l s r$ operon via inactivation of LsrR [25]. A recent study showed that phospho-AI-2 can directly bind to LsrR in vitro [29]. However, it remains unclear how phospho-AI-2 relieves the LsrR repression. In order to explore whether phospho-AI-2 affects LsrR binding to the $l s r R$ promoter in vitro, a series of gel shift assays were performed.

In vitro phosphorylation of the chemically synthesized AI-2 by LsrK was performed as described previously $[25,29]$. A full conversion of AI-2 to phospho-AI-2 was confirmed by high performance liquid chromatography (HPLC) assays. The quantity of phosphorylated AI-2 in the final preparation is almost the same as that of AI-2 we utilized before the start of the reaction, indicating that the conversion ratio is nearly $100 \%$. The phospho-AI- 2 was size-fractionated by using an ultrafiltration column to remove the LsrK protein. Gel shift assays were then performed with the purified LsrR protein and a molar excess of labeled DNA fragment from the $l s r R$ promoter. Phospho-AI-2 inhibits the binding of LsrR to the $l s r R$ promoter in a dose dependent manner, while AI-2 has no such effect (Figure 3A). From lane 2 to 5, the amount of the LsrR protein was equivalent, and when different amounts of AI-2 (the final concentration was increased from 0 to $390 \mu \mathrm{M}$ ) were added in the reaction mixtures, no reduction of the DNA-protein complex was observed.

A

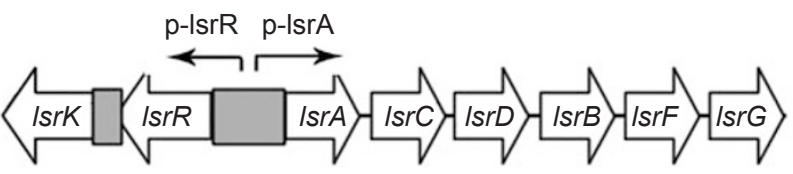

\section{B}
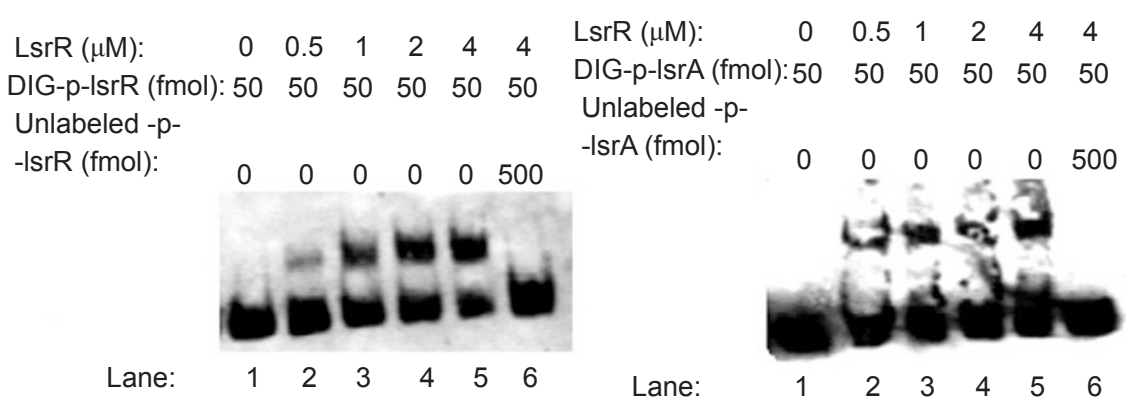

Figure 2 LsrR binds to the IsrA and IsrR promoters. (A) The IsrR gene is located immediately upstream of the IsrACDBFG operon and transcribed divergently, and the IsrA and IsrR promoters are located adjacent. Two DNA fragments p-IsrR (119 bp from -1 to -119 of the IsrR promoter region) and p-IsrA (129 bp from -1 to -129 of the IsrA promoter region) were amplified by PCR and DIG-labeled as probes, which are shown as arrows in the figure. (B) The binding ability of LsrR to the IsrR (the left one) and IsrA (the right one) promoters was determined by gel shift assays. Increasing amount of LsrR was incubated with excess DIG-labeled probes. In each panel, from lane 1 to 6 , the concentration of LsrR was $0,0.5,1,2,4$, and $4 \mu \mathrm{M}$, respectively; the amount of DIG-labeled probes was all the $50 \mathrm{fmol}$ (the concentrations were all $5 \mathrm{nM}$ ). In lane 6 , besides the labeled probes, $500 \mathrm{fmol}$ of unlabeled probes were incubated with the LsrR protein. 
By contrast, from lane 7 to 10, there are apparent reductions of the complexes with the addition of the different amounts of phospho-AI-2 (the final concentration of phospho-AI-2 was from 0 to $390 \mu \mathrm{M}$ ).

Since ADP exists as one of the products in the phospho-AI-2 preparation, and there may be residual ATP that was not converted to ADP during the in vitro phosphorylation reaction, we incubated ATP and ADP, respectively, with the LsrR protein and the DIG-labeled p-lsrR fragments under the same conditions. Our results show that ADP and ATP do not affect the binding of LsrR to the promoter regions (Figure 3B), ruling out the possibility that the above phenomenon was due to the presence of ADP or ATP. In summary, phospho-AI-2 renders LsrR unable to bind to the $l s r R$ promoter in in vitro assays.

\section{Identification of the LsrR-binding sites in the lsrR and lsrA promoters}

To further identify the precise LsrR-binding sequence, a DNase I footprinting was performed. A fragment incorporating the +51 to -119 region relative to the translation start site of the $l s r R$ gene was fluorescently 5 '-labeled with 6-carboxyfluorescein (6-FAM), and incubated with increasing amounts of $\mathrm{His}_{6}$-LsrR before being treated with DNase I. The digestion patterns were examined by capillary electrophoresis (CE) using a 3730XL automated sequencer (Applied Biosystems). The results of the CE were analyzed by Genemapper V3.5 software, through which the precise LsrR-binding sequence was obtained. As shown in Figure 4, one region from -20 to $-49 \mathrm{bp}$ is protected, which is indicated by the disappearing nucleotide peaks as the LsrR protein concentration increases (from line A to D). These footprinting results further demonstrated that LsrR binds to a 30-bp region (tga aca att gca tta aag att taa ata tgt) of its own promoter located just within the -35 to -10 promoter box relative to the translation start site (Figure 5A). By sequence alignment, we also found a 30-bp sequence (tca aaa ctc acc tgc aaa act gaa cgg ggg) from the $l s r A$ promoter region, which shared $66.7 \%$ identity with the p-lsrR-box. To assess whether it is also the LsrR-binding site, we carried out further gel shift assays. Two 30-bp DNA fragments (p-lsrR-box and p-lsrA-box) were DIG-labeled and used as the probes. As expected, both of them are able to form complexes upon the addition of 5 to 40 pmol of LsrR in a $10 \mu \mathrm{l}$ reaction mixture (Figure 5B).

Sequence analysis of LsrR-binding box and phenotypic assays of the mutated promoters of lsrR and lsrA

LsrR has been proposed to be a QS regulator that interacts with phospho-AI-2 and regulates transcription of a variety of genes in the previous studies [32], suggest-

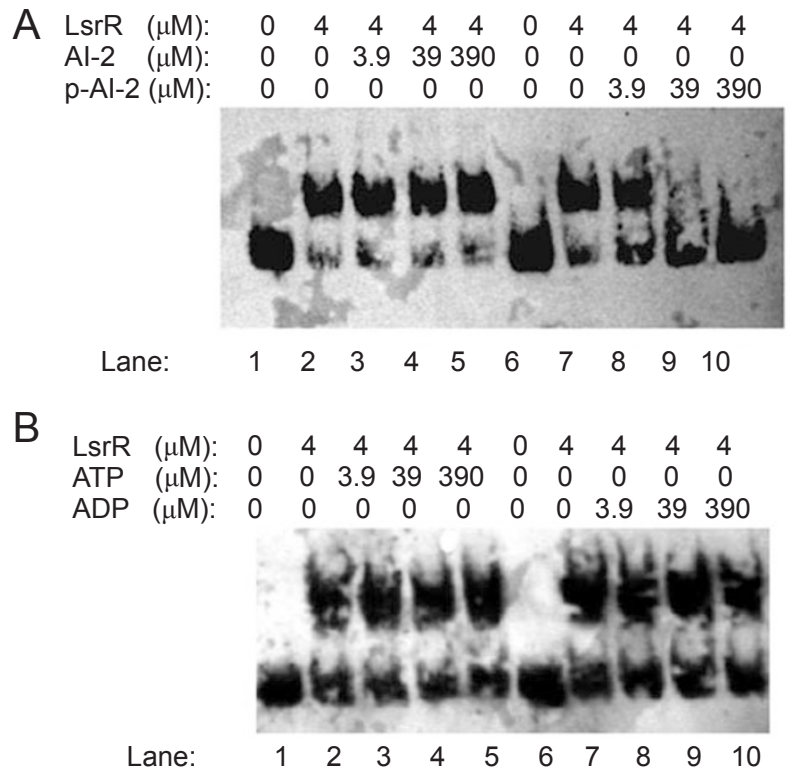

Figure 3 Phospho-Al-2 but not Al-2 causes LsrR to relieve its binding to the IsrR promoter. (A) Gel shift assays demonstrate that phospho-Al-2 causes LsrR to relieve its binding to the IsrR promoter. From lane 2 to 5 , the same amount of LsrR (the concentration was $4 \mu \mathrm{M}$ ) was incubated with $50 \mathrm{fmol}$ of labeled probes and increasing amount of $\mathrm{Al}-2$ (from lane 2 to 5 , the final concentration of Al-2 was $0,3.9,39$, and $390 \mu \mathrm{M}$, respectively); From lane 7 to 10 , the same amount of LsrR and labeled probes (similar with the above situation) was incubated with increasing amount of phospho-Al-2 (from lane 7 to 10, the final concentration of phospho-Al-2 was $0,3.9,39$, and $390 \mu \mathrm{M}$, respectively). Lane 1 and 6 were the controls. (B) Gel shift assays indicate that ADP or ATP in the phospho-Al-2 preparation has no effect on the binding ability of LsrR. ATP or ADP was incubated with the LsrR protein and the DIG-labeled p-IsrR fragments under the same conditions as shown in Figure $3 \mathrm{~A}$.

ing that LsrR might possess the ability to bind to diverse gene promoters. As mentioned above, the p-lsrR-box and p-lsrA-box do not share high similarity at the sequence level, indicating that the binding sites of LsrR are loosely conserved. To narrow down the sequences essential for LsrR binding, we performed a detailed analysis of the two binding boxes. By sequence alignment, we found that a 6-bp sequence (AAAACT in p-lsrA-box and AACAAT in p-lsrR-box) and a 9-bp sequence (AAAACTGAA in p-lsrA-box and AAGATTTAA in p-lsrR-box) are relatively conserved (Figure 6A), which led us to hypothesize that these two sequences might be crucial for LsrR binding. To test this, the $\beta$-galactosidase reporter plasmids pPlsrR and pPlsrA were used for assessing transcriptional activities of the $l s r A$ and $l s r R$ promoters [33]. We also constructed a series of derivatives of the above plasmids by making corresponding deletions (Figure 6B). 


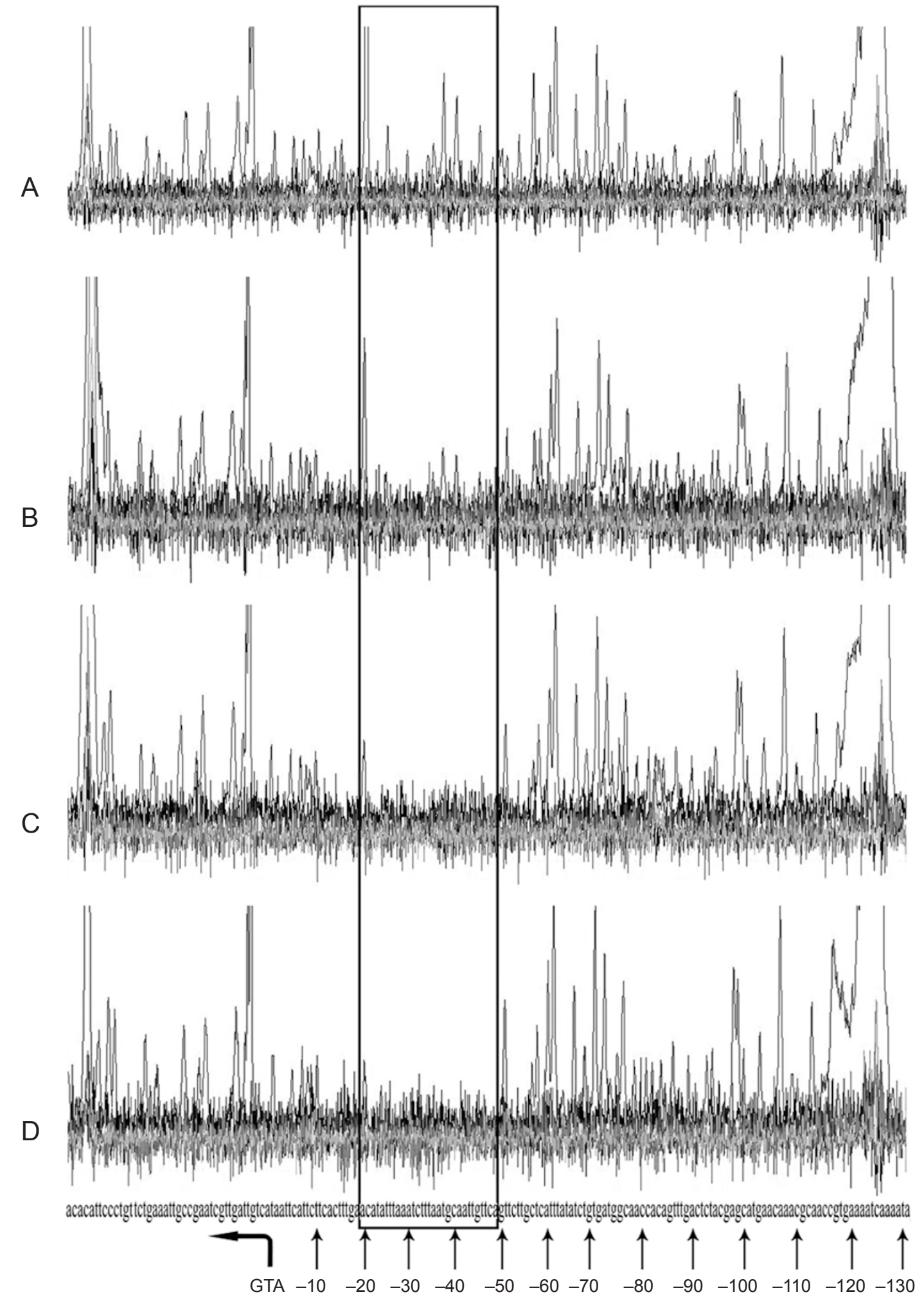

Figure 4 Identification of the LsrR-binding site to the promoter of the IsrR gene by DNase I footprinting assays. A fragment of the IsrR promoter region was PCR-amplified and 5'-FAM-labeled. DNase I digestion reactions were prepared and analyzed by capillary electrophoresis in an $\mathrm{ABI} 3730 \mathrm{XL}$ sequencer as described in experimental procedures. The black frame indicates the DNA region protected from DNase I by LsrR. Four electropherograms show the reactions with the following increasing concentration of LsrR: $0 \mu \mathrm{M}(\mathrm{A}), 2 \mu \mathrm{M}(\mathrm{B}), 4 \mu \mathrm{M}(\mathrm{C})$, and $8 \mu \mathrm{M}(\mathrm{D})$, respectively. 


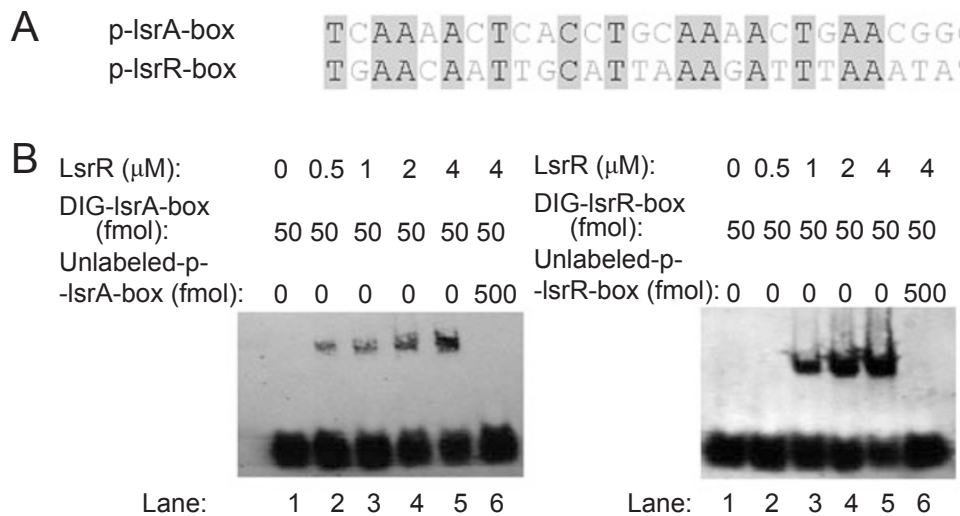

Figure 5 Nucleotide sequence alignment between the p-IsrR-box and the p-IsrA-box. (A) The alignment of the $p$-IsrR-box and p-IsrA-box was performed by using Vector NTI Suite 9 software. (B) The binding ability of LsrR to the p-IsrR-box and p-lsrAbox was determined by the gel shift assays. The fragments of the p-IsrR-box and p-lsrA-box were PCR-amplified and DIGlabeled as probes. Increasing amount of LsrR was incubated with excess DIG-labeled probes. In each panel, from lane 1 to 6 , the concentration of LsrR was $0,0.5,1,2,4$, and $4 \mu \mathrm{M}$, respectively; the amounts of DIG-labeled probes were all the 100 fmol. In lane 6 , besides the labeled probes, 1 pmol of unlabeled probes were incubated with the LsrR protein.
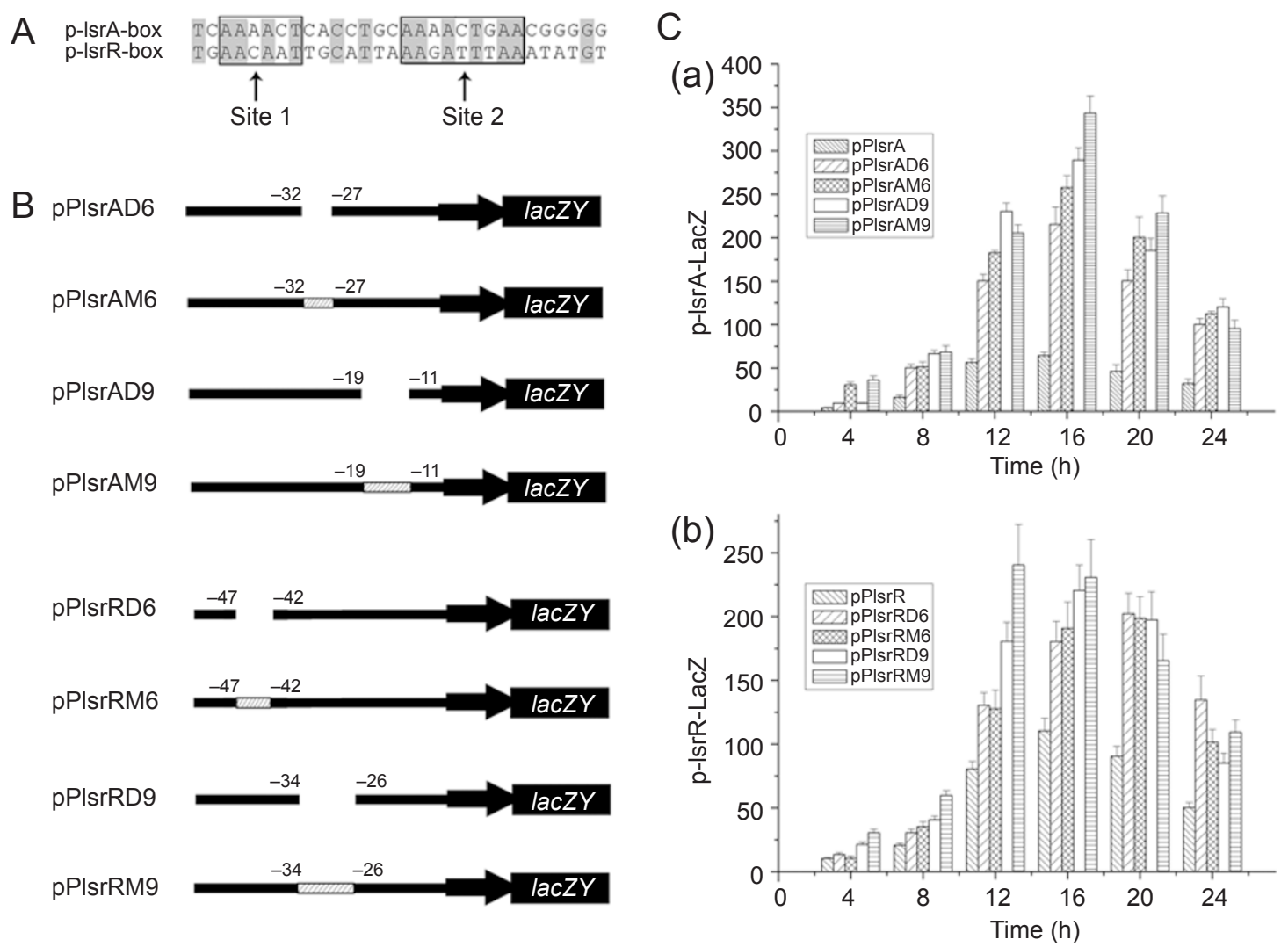

Figure 6 The conserved binding sequences of LsrR and the maps of the $\beta$-galactosidase reporter plasmids with mutated promoters. (A) The conserved binding sequences of LsrR were identified by sequence alignment between the $p$-lsrR-box and p-IsrA-box using Vector NTI Suite 9 software. The black frame indicates the regions of the conservative sites. (B) The various plasmids with IsrR and IsrA promoter fragments carrying 6- or 9-bp nucleotide bases deletions or mutations were derivatives of the plasmids pPIsrR and pPIsrA. The spaces represent deletions and the white squares represent random GC replacements. (C) $\beta$-galactosidase activity of the IsrR and IsrA promoters and their derivatives. $\beta$-galactosidase activities of the strains with corresponding plasmids were measured every $4 \mathrm{~h}$. Error bars indicate standard deviations. (a) E. coli DH5a containing plasmids pPIsrA, pPIsrAD6, pPIsrAM6, pPIsrAD9, and pPIsrAM9. (b) E. coli DH5a containing plasmids pPIsrR, pPIsrRD6, pPIsrRM6, pPIsrRD9, and pPIsrRM9. 
The above plasmids were transformed into E. coli DH5a (which is a luxS mutant strain, so that the transcriptional activities of $l s r A$ and $l s r R$ are repressed from the beginning to the end of the growth and cannot be activated by $\mathrm{AI}-2)$ and $\beta$-galactosidase activities were examined. The data in Figure 6C (a) show that, compared with pPlsrA, both pPlsrAD6 and pPlsrAD9 are associated with apparent increase in the expression of the LacZ reporter throughout the growth cycle. Correspondingly, as shown in Figure 6C (b), compared with pPlsrR, pPlsrRD6 and pPlsrRD9 are also associated with increase in the expression of the LacZ reporter. These results suggest that LsrR was unable to bind to the promoters of $l s r A$ and $l s r R$ firmly when the 6-bp or 9-bp sequences were deleted. Considering that deletion of 6 to 9 base-pair segments in such a binding site is likely to result in the reduction in binding affinity regardless of whether it included the exact binding motif or not, we constructed plasmids containing site-specific mutations (the original 6 to 9 base-pair AT-rich segments were replaced by random GCs) (Figure 6B), and conducted $\beta$-galactosidase activity assays after transformation of these mutant plasmids. As shown in Figure 6C, the transcriptional activities of p-lsrRM6, p-1srRM9, p-lsrAM6, and p-1srAM9 (similarly to that of p-lsrRD6, p-lsrRD9, p-lsrAD6, and p-lsrAD9) are all increased to higher levels compared with those of their parental promoters.

\section{Characteristics of the LsrR protein}

Three-dimensional structure prediction indicates that LsrR is composed of a typical HTH domain at the Nterminal and a sugar-ligand domain at the $\mathrm{C}$-terminal. We found that when overexpressed in vitro, the $\mathrm{C}$-terminal $\mathrm{His}_{6}$-tag LsrR protein mainly existed as tetramers rather than as monomers as shown by molecular sieve assays and the ratio was about 9:1 (data not shown). However, in the form of tetramer, LsrR still could bind to DNA probably because of the exposure of the N-terminal HTH domain. In vitro expressed $\mathrm{His}_{6}$-tag LsrR protein had the characteristic of strong binding affinity but weak stability, resulting in its quick denaturation and precipitation, thus bringing many challenges for the gel shift assays. Easy precipitation of the protein caused the DNA-protein complex to remain in the well and be unable to enter the gel. To solve the problem, many attempts including improvement of the protein purification methods and optimization of the experimental conditions were made. We found that the instability of the LsrR protein was mainly due to the instability of the N-terminal HTH domain. We had attempted to improve the stability of LsrR by adding the GST tag at its N-terminus, but it did make LsrR more stable and also dramatically decreased its DNA-binding affinity. It is likely that GST, due to its relatively large size $(27 \mathrm{kD})$, affected the conformation of the LsrR Nterminal, which is responsible for the DNA binding. At last, through improving experimental conditions to overcome the problem of easy precipitation of the protein, we were able to use the C-terminal $\mathrm{His}_{6}$-tag LsrR protein to obtain the above results. The optimization included some details in protein preparation and gel shift assays. First, expression of LsrR should be induced at $16{ }^{\circ} \mathrm{C}$; the purification should be performed at low temperature $\left(4^{\circ} \mathrm{C}\right)$ and high salt concentration $(1 \mathrm{M})$, and imidazole in the eluant should be removed by ultrafiltration rather than dialysis. Second, during gel shift assays, the binding reaction and electrophoresis need to be carried out at room temperature and $4{ }^{\circ} \mathrm{C}$, respectively; the samples should be loaded onto the well as quickly as possible, and the time of the electrophoresis should be within $1 \mathrm{~h}$.

\section{Discussion}

Bacteria have evolved complex mechanisms to regulate their physiological activities and behaviors in response to a variety of environmental cues. The AI-2dependent QS system is considered to be the main QS system in E. coli and S. typhimurium, and many details about the mechanisms of this system still need to be further investigated. In S. typhimurium and E. coli, the AI-2 QS system regulates gene expression in a cytoplasmic AI-2-dependent manner. However, as the derivative of a furan ring, neither AI-2 nor phospho-AI-2 can directly interact with a target gene and function as a direct regulator to control gene transcription. The LsrR protein, a member of the transcription factor family based on the structural prediction, most probably acts as the important connector linking the detection of AI-2 to the regulation of gene expression $[25,28,29,32]$. The role of LsrR as a transcriptional regulator that responds to the QS signal AI-2 has been determined in the previous studies. Meanwhile, a greater impact of LsrR on gene expression control has been revealed. According to a previous study, 146 genes are significantly affected by the $l s r R$ deletion. This includes genes related to secretion systems, biofilm formation, and genes coding for transcriptional regulators, small RNAs or riboregulators, and regulatory proteins for stress responses and nutrient acquisition [32]. LsrR has even been hypothesized to be a global QS regulator that acts in tandem with unphosphorylated AI-2 or its anomer to regulate the transcription of a variety of genes. No matter whether this hypothesis is true or not, the regulatory mode of LsrR will be a topic of great interest. In this study, we performed experiments to study the binding characteristics of LsrR to the promoter 
regions and performed detailed analysis of the binding sequences, providing clues for the prediction of the target genes modulated by LsrR and illuminating the relationship between LsrR and the genes regulated by the AI-2 QS system.

A previous study has demonstrated that LsrR can bind to phospho-AI-2 in vitro, the effective form of the QS signal, indicating that LsrR is capable of signal detection [29]. However, as a widely predicted transcription factor, LsrR has not been shown to directly bind to any DNA fragment so far. Previous physiological studies showed that LsrR represses the transcription of $l s r$ operon and itself, but did not reveal whether the effects are through direct or indirect interactions between LsrR and these promoters. Our present study has provided direct evidence for the DNA-binding ability of LsrR, and confirmed that LsrR represses the transcription of $l s r$ operon and itself by directly binding to their promoter regions.

The study of the LsrR-binding sequences in the promoter regions ( $\mathrm{p}-\mathrm{lsrA}$ and $\mathrm{p}-\mathrm{lsrR}$ ) shows that both on $\mathrm{p}$ lsrA and p-lsrR, there exists a 30-bp binding box, which can be recognized by LsrR. The sequences are from -20 to $-49 \mathrm{bp}$ relative to the translation start codon of $l s r R$ and from -5 to -34 bp relative to the translation start codon of $l s r A$. Interestingly, these regions represent the usual binding sites of transcription factors or the transcriptional machinery, such as $\sigma$ factor and RNA poly- merase. We propose that the effect of LsrR repression is due to its competitive inhibition of these transcriptional factors, thus making them unable to bind to the promoter regions. By comparing the two 30-bp binding boxes, we found that the similarity between the p-lsrR-box and the p-lsrA-box is not high at the nucleotide level (Figure $5 \mathrm{~A}$ ), indicating that the LsrR-binding sequences do not need to share high homology. However, we found two highly homologous motifs within the two binding boxes: AAAACTNN---NNAAAACTGAA in the p-lsrA-box and AACAATNN---NNAAGATTTAA in the p-lsrR-box, and the motifs contain high ratios of $\mathrm{A}$ and $\mathrm{T}$. Results of the in vivo physiological experiments further revealed that either deletion or mutation of these motifs resulted in an apparent increase of the transcriptional activity of the promoters. We used E. coli DH5 $\alpha$, a LuxS/AI-2-negative strain as the host to test transcriptional activities of the promoters through measuring the $\beta$-galactosidase reporter activities. Our data showed that the transcriptional activities of the parental p-lsrR and p-lsrA remained at low levels throughout the growth cycle because of the LsrR repression; in contrast, the transcriptional activities of the p-lsrR and p-lsrA mutants with 6- to 9-bp deletions or random GC replacement increased to higher levels. These data indicate that the above motifs might be essential for LsrR binding. In addition, high ratios of A and $\mathrm{T}$ within these motifs suggest that LsrR might preferen-

Table 1 Strains and plasmids used in this study

\begin{tabular}{|c|c|c|}
\hline Strain or plasmid & Relevant genotype or characteristics & Reference or source \\
\hline \multicolumn{3}{|l|}{ E. coli strains } \\
\hline MG1655 & Wild type & Laboratory stock \\
\hline $\mathrm{DH} 5 \alpha$ & $\begin{array}{l}\text { Clone host strain, luxS mutant, supE444lacU169 ( } \varphi 80 \text { lacZ } \Delta \mathrm{M} 15) \text { hsdR17 recAlen- } \\
\text { dA1 gyrA96 thi-1 relA1 }\end{array}$ & Laboratory stock \\
\hline BL21 (DE3) & $F, o m p T, h s d S\left(r B^{-} m B^{-}\right), g a l, d c m(\mathrm{DE} 3)$ & Laboratory stock \\
\hline \multicolumn{3}{|l|}{ Plasmids } \\
\hline $\mathrm{pET} 28 \mathrm{a}(+)$ & Expression vector with hexahistidine tag, $\mathrm{Kan}^{\mathrm{r}}$ & Novagen \\
\hline pGlsrR & pET28a(+) with $l s r R, \operatorname{Kan}^{\mathrm{r}}$ & This study \\
\hline pGlsrK & pET28a $(+)$ with $l s r K, \operatorname{Kan}^{\mathrm{r}}$ & This study \\
\hline pPlsrR & pFZY1 derivative, LacZ-reporter plasmid, containing lsr $R$ promoter region, $\mathrm{Amp}^{\mathrm{r}}$ & Zhou X et al. [33] \\
\hline pPlsrA & pFZY1 derivative, LacZ-reporter plasmid, containing $l s r A$ promoter region, Amp ${ }^{\mathrm{r}}$ & Zhou X et al. [33] \\
\hline pPlsrRD6 & Mutational pPlsrR with 6-bp 'AACAAT' deletion & This study \\
\hline pPlsrRD9 & Mutational pPlsrR with 9-bp 'AAGATTTAA' deletion & This study \\
\hline pPlsrAD6 & Mutational pPlsrA with 6-bp 'AAAACT' deletion & This study \\
\hline pPlsrAD9 & Mutational pPlsrA with 9-bp 'AAAACTGAA' deletion & This study \\
\hline pPlsrRM6 & Mutational pPlsrR with 6-bp mutation ('AACAAT’ to 'GCGCGC’) & This study \\
\hline pPlsrRM9 & Mutational pPlsrR with 9-bp mutation ('AAGATTTAA' to 'GCGCGGCCC') & This study \\
\hline pPlsrAM6 & Mutational pPlsrA with 6-bp mutation ('AAAACT’ to 'GCGCGC') & This study \\
\hline pPlsrAM9 & Mutational pPlsrA with 9-bp mutation ('AAAACTGAA' to 'GCGCGGCCC') & This study \\
\hline
\end{tabular}


tially bind to AT-rich sequences.

\section{Materials and Methods}

\section{Bacterial strains and media}

The bacterial strains and plasmids used in this study are listed in Table 1. All strains were grown in Luria broth (LB), which contained $10 \mathrm{~g} / 1$ Bacto tryptone (Oxoid), $5 \mathrm{~g} / 1$ yeast extract (Oxoid), and $10 \mathrm{~g} / 1 \mathrm{NaCl}$. When necessary, media were supplemented with antibiotics at the following concentrations $(\mathrm{mg} / 1)$ : ampicillin (Amp), 100; kanamycin (Kan), 50. All E. coli strains were grown at $37^{\circ} \mathrm{C}$ with aeration.

\section{Plasmid construction}

To construct plasmids pGlsrR and pGlsrK used for the overproduction of LsrR and LsrK, the DNA fragment (954 bp) containing the $l s r R$ coding region and the DNA fragment (1 $593 \mathrm{bp}$ ) containing the $l s r K$ coding region were amplified by polymerase chain reaction (PCR) from the chromosome of E. coli MG1655, respectively, using two pairs of primers (g-lsrR-f and g-lsrR-r; glsrK-f and g-lsrK-r). The PCR products were digested with NcoI and XhoI, and subsequently were ligated with NcoI and XhoIdigested pET28a $(+)$ (Novagen). The inserts were checked by DNA sequencing. Plasmids $\mathrm{pPlsrR}$ and $\mathrm{pPlsrA}$ were the $\beta$-galactosidase reporter plasmids used for measuring the transcriptional activity of the $l s r A$ and $l s r R$ promoters [33]. Plasmids pPlsrRD6, pPlsrRM6, pPlsrRD9, pPlsrRM9, pPlsrAD6, pPlsrRM6, pPlsrAD9, and pPlsrAD9 were the plasmids with mutated promoter regions (6- or 9-bp nucleotide bases deletion or mutation) of pPlsrR and pPlsrA (Figure 6B). To create them, the method of site-directed mutagenesis by PCR [34, 35] was used and modified. For example, consider pPlsrRD6: there were 6-bp nucleotide bases (AACAAT) in the $l s r R$ promoter region, which were proposed to be necessary for LsrR binding, the 6-bp nucleotide bases were deleted from pPlsrA by the method as follows: a DNA fragment (about $10.5 \mathrm{~kb}$, containing the full length of the plasmid except the 6-bp nucleotide bases) was amplified by PCR using the pPlsrR as the template, and D6-p-lsrR-f and D6-p-lsrR-r as the primers and Primer Star DNA polymerase (Takara). The PCR product was digested with DpnI (New England Biolabs), and then the digested product was phosphorylated, self-ligated, and transformed into E. coli DH5 $\alpha$. The positive clones with mutational promoter regions were checked by DNA sequencing. The sequences of all primers used in this study are listed in Table 2.

\section{Purification of LsrR and LsrK}

The same purification methods were used for LsrR and LsrK. Plasmid was transformed into E. coli BL21 (DE3). The transformant was grown in $1 \mathrm{~L}$ of $\mathrm{LB}$ at $37^{\circ} \mathrm{C}$ to an $\mathrm{OD}_{600}$ of 0.4 , transferred to $16^{\circ} \mathrm{C}$, and induced overnight with $0.1 \mathrm{mM}$ IPTG. Cells were harvested by centrifugation and washed with cell washing buffer (20 mM Tris-HCl, pH 8.0 and $1 \mathrm{M} \mathrm{NaCl}$ ). The cells were resuspended in $50 \mathrm{ml}$ of lysis buffer $(20 \mathrm{mM}$ Tris- $\mathrm{HCl}, \mathrm{pH} 8.0$ and $1 \mathrm{M} \mathrm{NaCl}$ ) and were then lysed by sonication, and centrifuged at $12500 \mathrm{rpm}$ for $30 \mathrm{~min}$ at $4{ }^{\circ} \mathrm{C}$. The supernatant was mixed with 2 $\mathrm{ml}$ of Ni-NTA agarose solution (Invitrogen), and the suspension was loaded onto a column at $4{ }^{\circ} \mathrm{C}$. After washing the column with $5 \mathrm{ml}$ of washing buffer I ( $5 \mathrm{mM}$ imidazole, $20 \mathrm{mM}$ Tris- $\mathrm{HCl}, \mathrm{pH}$ 8.0 , and $1 \mathrm{M} \mathrm{NaCl})$, and then with $100 \mathrm{ml}$ of washing buffer II (20
Table 2 Oligonucleotide primers used in this study

\begin{tabular}{|c|c|}
\hline Primer name & Oligonucleotide $\left(5^{\prime}-3^{\prime}\right)^{\mathrm{a}}$ \\
\hline g-lsrR-f & GCGccatggCAATCAACGATTCGGC \\
\hline g-lsrR-r & GCGctcgagACTACGTAAAATCGCCGCTG \\
\hline g-lsrK-f & GCGecatggCTCGACTCTTTACCCTTTC \\
\hline g-lsrK-r & GCGctegagTAACCCAGGCGCTTTCCATA \\
\hline p-lsrR-f & AАTTCATTCTTCACTTTGAACAT \\
\hline p-lsrR-r & TTATGCTATTTTGATTTTCACGG \\
\hline p-lsrA-f & ATTGTGATCTATTCGTCGGAAATAT \\
\hline p-lsrA-r & ATTTCCCCCGTTCAGTTTTG \\
\hline p-lsrR-f-FAM & TTCTTCTTCACACATTCCCTGT \\
\hline D6-p-lsrR-f & CAGTTCTTGCTCATTTATATC \\
\hline D6-p-1srR-r & TGCATTAAAGATTTAAATATG \\
\hline D9-p-lsrR-f & TAATGCAATTGTTCAGTTCT \\
\hline D9-p-lsrR-r & ATATGTTCAAAGTGAAGAATG \\
\hline D6-p-lsrA-f & CACCTGCAAAACTGAACGG \\
\hline D6-p-lsrA-r & GAACAAATGTATTTCTGCTTTT \\
\hline D9-p-lsrA-f & CGGGGGAAATATGCAAACGAG \\
\hline D9-p-lsrA-r & GCAGGTGAGTTTTGAACAAATG \\
\hline M6-p-lsrR-f & CAGTTCTTGCTCATTTATATC \\
\hline M6-p-lsrR-r & GCGCGCTGCATTAAAGATTTAAATATG \\
\hline M9-p-lsrR-f & GGCCTAATGCAATTGTTCAGTTCT \\
\hline M9-p-1srR-r & GCGCGATATGTTCAAAGTGAAGAATG \\
\hline M6-p-lsrA-f & CACCTGCAAAACTGAACGG \\
\hline M6-p-lsrA-r & GCGCGCGAACAAATGTATTTCTGCTTTT \\
\hline M9-p-lsrA-f & GGCCCGGGGGAAATATGCAAACGAG \\
\hline M9-p-lsrA-r & GCGCGGCAGGTGAGTTTTGAACAAATG \\
\hline
\end{tabular}

${ }^{\mathrm{a}}$ The sequences in bold lowercase letters refer to the restriction endonuclease recognition sites.

$\mathrm{mM}$ imidazole, $20 \mathrm{mM}$ Tris- $\mathrm{HCl}, \mathrm{pH} 8.0$, and $1 \mathrm{M} \mathrm{NaCl})$ and 10 $\mathrm{ml}$ washing buffer III (100 mM imidazole, $20 \mathrm{mM}$ Tris-HCl, pH 8.0, and $1 \mathrm{M} \mathrm{NaCl}$ ), the LsrR protein was eluted with $5 \mathrm{ml}$ of elution buffer $(250 \mathrm{mM}$ imidazole, $20 \mathrm{mM}$ Tris-HCl, $\mathrm{pH} 8.0,1 \mathrm{M} \mathrm{NaCl}$, and $10 \%$ glycerol). The imidazole in the eluant was removed by using a Centrifuge Biomax-5 column (Millipore), and then the LsrR protein solution was stored at $-80{ }^{\circ} \mathrm{C}$ until use. The purity of the protein was analyzed by SDS-PAGE and the protein concentration was measured using the Bradford assay with bovine serum albumin (BSA) as a standard.

\section{Gel shift assay}

Two DNA fragments p-lsrA (129 bp) containing lsr A promoter and $\mathrm{p}-1 \mathrm{srR}(119 \mathrm{bp})$ containing $l s r R$ promoter were prepared by PCR from chromosome of $E$. coli MG1655 using two pairs of primers (p-lsrA-f, p-lsrA-r, p-lsrR-f, and p-lsrR-r) and pfu DNA polymerase (Promega). The PCR products were purified by polyacrylamide gel electrophoresis (PAGE). The fragments p-lsrA-box (30 bp) and p-lsrR-box (30 bp) were synthesized by the manufacturer (Invitrogen). A digoxigenin (DIG) gel shift kit (second generation, Roche) was used for labeling of DNA fragments and 
detection of signals according to the manufacturer's instructions. Binding reactions were performed by incubating the labeled DNA fragments with various amounts of purified LsrR at $25^{\circ} \mathrm{C}$ for 10 $15 \mathrm{~min}$ in $10 \mu \mathrm{l}$ of incubation buffer $(50 \mathrm{mM}$ Tris- $\mathrm{HCl}, \mathrm{pH} 7.5$, $100 \mathrm{mM} \mathrm{NaCl}, 3 \mathrm{mM}$ magnesium acetate, $0.1 \mathrm{mM}$ EDTA, and 0.1 $\mathrm{mM}$ dithiothreitol (DTT)). After the incubation, the mixtures were added with $2.5 \mu \mathrm{l}$ of gel loading buffer $(0.25 \times \mathrm{TBE}, 60 \%$; glycerol, $40 \%$; and bromphenol, $0.2 \%$ (wt/vol)), and then were electrophoresed in a $4 \%$ native polyacrylamide gel in $0.5 \times$ TBE buffer $(45$ $\mathrm{mM}$ Tris-borate and $1 \mathrm{mM}$ EDTA; $\mathrm{pH}$ 8.0). DNA bands were detected and analyzed according to the manufacturer's instructions.

\section{DNase I footprinting assay}

PCR primer was synthesized and subsequently 5'-labeled with 6-FAM by the manufacturer (Invitrogen), resulting in the labeled primer p-lsrR-f-FAM. The labeled DNA fragments were prepared by PCR using E. coli MG1655 genomic DNA as template, labeled p-lsrR-f-FAM, and unlabeled p-lsrR-r as primers. The labeled DNA fragments were purified by PAGE. DNase I footprinting assays were performed with a 3730XL DNA analyzer (Applied Biosystems) using a modified method based on previous studies [36, 37]. For DNA footprinting assay, the labeled promoter fragments ( $1 \mathrm{pmol}$ ) were incubated with purified LsrR in $50 \mu \mathrm{l}$ of incubation buffer (50 mM Tris- $\mathrm{HCl}, \mathrm{pH} 7.5,150 \mathrm{mM} \mathrm{NaCl}, 3 \mathrm{mM}$ magnesium acetate, $0.1 \mathrm{mM}$ EDTA, and $0.1 \mathrm{mM}$ DTT) for $20 \mathrm{~min}$ at the room temperature; subsequently, the $\mathrm{Ca}^{2+} / \mathrm{Mg}^{2+}$ solution $(50 \mu \mathrm{l}$, $5 \mathrm{mM} \mathrm{CaCl}_{2}$ and $10 \mathrm{mM} \mathrm{MgCl}_{2}$ ) was added into the mixture to a final volume of $100 \mu \mathrm{l}$ and then were incubated for $5 \mathrm{~min}$ at $25^{\circ} \mathrm{C}$. DNA digestion was initiated by the addition of $0.6 \mathrm{U}$ RQ1 RNaseFree DNase I (Promega). After digestion for $1 \mathrm{~min}$, the digest reaction was terminated by adding with $90 \mu \mathrm{l}$ stop solution (200 $\mathrm{mM} \mathrm{NaCl}, 30 \mathrm{mM}$ EDTA, and 1\% SDS). The final reaction mixtures then were extracted with $200 \mu \mathrm{l}$ phenol-chloroform-isoamyl alcohol $(25: 24: 1)$ and purified by using the ethanol precipitation method. Samples were loaded onto a 3730XL DNA analyzer (Applied Biosystems) for $\mathrm{CE}$ and detection. Electropherograms were aligned using GeneMapper 3.5 (Applied Biosystems).

\section{In vitro phosphorylation of AI-2}

In vitro phosphorylation assays were performed with the methods as described previously [26, 29]. The reactions were performed in $2 \mathrm{ml}$ mixtures containing $1 \mathrm{mg}$ of purified His-tagged LsrK, 25 mM Hepes, $\mathrm{pH} 7.5,0.1 \mathrm{mg} / \mathrm{ml} \mathrm{BSA}$, and $1 \mathrm{mM}$ DTT. Each sample contained $2 \mathrm{mM}$ cold ATP, $100 \mathrm{mM} \mathrm{MgCl}$, and $1.3 \mathrm{mM} \mathrm{AI-2} \mathrm{(DPD,}$ Omm.Scientific Inc.). Reaction mixtures were incubated at $37{ }^{\circ} \mathrm{C}$ for $3 \mathrm{~h}$, and then the phospho-AI-2 was size-fractionated by using a Centrifuge Biomax-5 column (Millipore) to remove the LsrK protein. Serial dilutions were applied to HPLC analysis (Agilent). In the phospho-AI-2 preparation, phosphorylated AI-2 is in a $1: 1$ molar ratio with ADP. Quantification of phosphorylated AI-2 was performed by measuring the concentration of ADP by HPLC (standard curve method). The conversion efficiency is the ratio of the quantity of phosphorylated AI-2 in the final preparation and that of AI-2 we utilized before the start of the reaction. Freshly prepared ATP and ADP stock solutions of known concentrations were used to draw the standard curves and confirm the accuracy of this method.

\section{$\beta$-galactosidase assay}

Stationary phase cultures of E. coli DH5 $\alpha$ were diluted 1:500 into $20 \mathrm{ml}$ of $\mathrm{LB}$ with $100 \mu \mathrm{g} / \mathrm{ml}$ ampicillin and grown for the specified times. After growth, cells were harvested and resuspended in $1 \mathrm{ml}$ of $\mathrm{Z}$ buffer, and $\beta$-galactosidase assays were performed as described previously [38]. $\beta$-Galactosidase units are defined as $\left(\mathrm{OD}_{420} \times 1000\right) /\left(\mathrm{OD}_{600} \times\right.$ Volume $(\mathrm{ml}) \times$ Time $\left.(\mathrm{min})\right)$. All assays are reported as the mean $\beta$-galactosidase activity of three independent cultures, and error bars represent the standard deviations.

\section{Acknowledgments}

We thank our colleagues J Zang and X Liu for their technical assistance in protein purification. This work was supported by the One Hundred Talent Project of the Chinese Academy of Sciences and the National Natural Science Foundation of China (50738006).

\section{References}

1 Bassler BL. How bacteria talk to each other: regulation of gene expression by quorum sensing. Curr Opin Microbiol 1999; 2:582-587.

2 Miller MB, Bassler BL. Quorum sensing in bacteria. Annu Rev Microbiol 2001; 55:165-199.

3 Ahmer BM. Cell-to-cell signalling in Escherichia coli and Salmonella enterica. Mol Microbiol 2004; 52:933-945.

4 Bassler BL, Wright M, Silverman MR. Multiple signalling systems controlling expression of luminescence in Vibrio harveyi: sequence and function of genes encoding a second sensory pathway. Mol Microbiol 1994; 13:273-286.

5 De Keersmaecker SC, Sonck K, Vanderleyden J. Let LuxS speak up in AI-2 signaling. Trends Microbiol 2006; 14:114119.

6 Federle MJ, Bassler BL. Interspecies communication in bacteria. J Clin Invest 2003; 112:1291-1299.

7 Ohtani K, Hayashi H, Shimizu T. The luxS gene is involved in cell-cell signalling for toxin production in Clostridium perfringens. Mol Microbiol 2002; 44:171-179.

8 Sun J, Daniel R, Wagner-Dobler I, Zeng AP. Is autoinducer-2 a universal signal for interspecies communication: a comparative genomic and phylogenetic analysis of the synthesis and signal transduction pathways. BMC Evol Biol 2004; 4:36.

9 Surette MG, Miller MB, Bassler BL. Quorum sensing in Escherichia coli, Salmonella typhimurium, and Vibrio harveyi: a new family of genes responsible for autoinducer production. Proc Natl Acad Sci USA 1999; 96:1639-1644.

10 Vendeville A, Winzer K, Heurlier K, Tang CM, Hardie KR. Making 'sense' of metabolism: autoinducer-2, LuxS and pathogenic bacteria. Nat Rev Microbiol 2005; 3:383-396.

11 Bassler BL, Wright M, Silverman MR. Sequence and function of LuxO, a negative regulator of luminescence in Vibrio harveyi. Mol Microbiol 1994; 12:403-412.

12 Davies DG, Parsek MR, Pearson JP, et al. The involvement of cell-to-cell signals in the development of a bacterial biofilm. Science 1998; 280:295-298.

13 Merritt J, Qi F, Goodman SD, Anderson MH, Shi W. Mutation of luxS affects biofilm formation in Streptococcus mutans. Infect Immun 2003; 71:1972-1979.

14 Sperandio V, Mellies JL, Nguyen W, Shin S, Kaper JB. Quorum sensing controls expression of the type III secretion gene transcription and protein secretion in enterohemorrhagic and 
enteropathogenic Escherichia coli. Proc Natl Acad Sci USA 1999; 96:15196-15201.

15 Bassler BL, Wright M, Showalter RE, Silverman MR. Intercellular signalling in Vibrio harveyi: sequence and function of genes regulating expression of luminescence. Mol Microbiol 1993; 9:773-786.

16 Winzer K, Hardie KR, Burgess N, et al. LuxS: its role in central metabolism and the in vitro synthesis of 4-hydroxy-5methyl-3(2H)-furanone. Microbiology 2002; 148 (Part 4):909922.

17 Chen X, Schauder S, Potier N, et al. Structural identification of a bacterial quorum-sensing signal containing boron. Nature 2002; 415:545-549.

18 Li J, Wang L, Hashimoto Y, et al. A stochastic model of Escherichia coli AI-2 quorum signal circuit reveals alternative synthesis pathways. Mol Syst Biol 2006; 2:67.

19 Miller ST, Xavier KB, Campagna SR, et al. Salmonella typhimurium recognizes a chemically distinct form of the bacterial quorum-sensing signal AI-2. Mol Cell 2004; 15:677-687.

20 Schauder S, Shokat K, Surette MG, Bassler BL. The LuxS family of bacterial autoinducers: biosynthesis of a novel quorum-sensing signal molecule. Mol Microbiol 2001; 41:463476.

21 Semmelhack MF, Campagna SR, Federle MJ, Bassler BL. An expeditious synthesis of DPD and boron binding studies. Org Lett 2005; 7:569-572.

22 Waters CM, Bassler BL. Quorum sensing: cell-to-cell communication in bacteria. Annu Rev Cell Dev Biol 2005; 21:319346.

23 Lenz DH, Mok KC, Lilley BN, et al. The small RNA chaperone Hfq and multiple small RNAs control quorum sensing in Vibrio harveyi and Vibrio cholerae. Cell 2004; 118:69-82.

24 Lilley BN, Bassler BL. Regulation of quorum sensing in Vibrio harveyi by LuxO and sigma-54. Mol Microbiol 2000; 36:940-954.

25 Taga ME, Miller ST, Bassler BL. Lsr-mediated transport and processing of AI-2 in Salmonella typhimurium. Mol Microbiol 2003; 50:1411-1427.

26 Taga ME, Semmelhack JL, Bassler BL. The LuxS-dependent autoinducer AI-2 controls the expression of an ABC transporter that functions in AI-2 uptake in Salmonella typhimurium.
Mol Microbiol 2001; 42:777-793.

27 Wang L, Hashimoto Y, Tsao CY, Valdes JJ, Bentley WE. Cyclic AMP (cAMP) and cAMP receptor protein influence both synthesis and uptake of extracellular autoinducer 2 in Escherichia coli. J Bacteriol 2005; 187:2066-2076.

28 Xavier KB, Bassler BL. Interference with AI-2-mediated bacterial cell-cell communication. Nature 2005; 437:750-753.

29 Xavier KB, Miller ST, Lu W, et al. Phosphorylation and processing of the quorum-sensing molecule autoinducer-2 in enteric bacteria. ACS Chem Biol 2007; 2:128-136.

30 Xavier KB, Bassler BL. Regulation of uptake and processing of the quorum-sensing autoinducer AI-2 in Escherichia coli. $J$ Bacteriol 2005; 187:238-248.

31 Wang L, Li J, March JC, Valdes JJ, Bentley WE. luxSdependent gene regulation in Escherichia coli $\mathrm{K}-12$ revealed by genomic expression profiling. J Bacteriol 2005; 187:83508360 .

32 Li J, Attila C, Wang L, et al. Quorum sensing in Escherichia coli is signaled by AI-2/LsrR: effects on small RNA and biofilm architecture. J Bacteriol 2007; 189:6011-6020.

33 Zhou X, Meng X, Sun B. An EAL domain protein and cyclic AMP contribute to the interaction between the two quorum sensing systems in Escherichia coli. Cell Res 2008; 18:937948.

34 Giebel LB, Spritz RA. Site-directed mutagenesis using a double-stranded DNA fragment as a PCR primer. Nucleic Acids Res 1990; 18:4947.

35 Kammann M, Laufs J, Schell J, Gronenborn B. Rapid insertional mutagenesis of DNA by polymerase chain reaction (PCR). Nucleic Acids Res 1989; 17:5404.

36 Yindeeyoungyeon W, Schell MA. Footprinting with an automated capillary DNA sequencer. Biotechniques 2000; 29:1034-1036, 1038, 1040-1041.

37 Zianni M, Tessanne K, Merighi M, Laguna R, Tabita FR. Identification of the DNA bases of a DNase I footprint by the use of dye primer sequencing on an automated capillary DNA analysis instrument. J Biomol Tech 2006; 17:103-113.

38 Slauch JM, Silhavy TJ. cis-acting ompF mutations that result in OmpR-dependent constitutive expression. J Bacteriol 1991; 173:4039-4048. 